\title{
Novel combination immunochemotherapy beyond CD20 for B-cell lymphomas
}

\author{
Jun Ho Yi \\ Division of Hematology-Oncology, Department of Medicine, Chung-Ang University Hospital, Seoul, Korea
}

p-ISSN 2287-979X / e-ISSN 2288-0011 https://doi.org/10.5045/br.2021.2020320

Blood Res 2021;56:S1-S4.

Received on December 13, 2020

Revised on February 16, 2021

Accepted on March 5, 2021

\author{
Correspondence to \\ Jun Ho Yi, M.D., Ph.D. \\ Division of Hematology-Oncology, \\ Department of Medicine, Chung-Ang \\ University Hospital, 102 Heukseok-ro, \\ Dongjak-gu, Seoul 06973, Korea \\ E-mail: xuno@daum.net \\ (C) 2021 Korean Society of Hematology
}

\begin{abstract}
Despite substantially improved survival with rituximab-based treatment regimens, there is an unmet medical need for better treatments of B-cell lymphoma, particularly for patients with relapsed or refractory disease. Retreatment with rituximab exerts a limited effect in these patients, and platinum-based salvage treatment followed by autologous stem cell transplantation remains the only curative option. Recent strategies have focused on targeting novel B-cell surface markers, inhibiting B-cell receptor signaling, and enhancing the cytotoxicity of effector cells. The current article will review the recent progress in immunochemotherapy targeting other than CD20 for B-cell lymphomas.
\end{abstract}

Key Words B-cell lymphoma, CD19, CD22, CD79b

\section{INTRODUCTION}

B-cell lymphomas can develop from different developmental stages of B cells in the germinal centers of secondary lymphoid tissue. The evolution of B-cell lymphomagenesis depends on a number of signaling pathways and proteins that play a key role in signaling networks and can be altered by various chromosomal translocations, genetic amplifications, mutations, and deletions [1]. Among the proteins that are involved in B-cell lymphomagenesis, CD20 has been an attractive target for several reasons, including that it presents in over $95 \%$ of mature B-cell neoplasms, and the facts that it is not shed from the surface after antibody binding, nor is it internalized or downregulated upon antibody binding [2]. Following the approval of rituximab in 1997 and obinutuzumab in 2013 by the US Food and Drug Administration (FDA), the targeting of CD20 has played a pivotal role in the treatment of B-cell lymphoma, and substantial improvements in survival outcomes have been observed [3, 4]. Despite these achievements, there has been an area of unmet medical needs, particularly for high-risk patients and patients with relapsed or refractory (RR) disease [5-7]. Until recently, a number of rituximab- or obinutuzumab-based variations have been explored to overcome anti-CD20 treat- ment failure, with limited success [8-10], and once rituximab-based treatment fails, re-treatment with rituximab has limited activity [11].

Thanks to recent advances in genetic engineering, immunotherapeutic agents other than 'naked' monoclonal antibodies, including recombinant immunotoxin (fragment of monoclonal antibody plus protein toxin), antibody-drug conjugates (monoclonal antibody plus cytotoxic drug), and bispecific antibodies (an artificial protein that can simultaneously bind to two different types of antigens, both B-cell and T-cell markers), have been used for the treatment of B-cell lymphomas.

In the current article, I will briefly review the recent progress in immunochemotherapy against targets other than CD20 for B-cell lymphomas.

\section{Targeting CD30}

CD30 is a pathognomonic marker of Hodgkin's lymphoma and anaplastic large cell lymphoma, but its expression is also detectable in B-cell lymphomas, including diffuse large B-cell lymphoma (DLBCL) $[12,13]$ and primary mediastinal large B-cell lymphoma [14, 15].

Brentuximab vedotin (BV), an ADC targeting CD30, was administered to patients with RR CD30-positive B-cell lymphomas, including 65 patients with DLBCL [16]. Single-agent 
BV produced an overall response rate (ORR) of $44 \%$, and the addition of rituximab showed an ORR of $46 \%$. BV in combination with R-CHOP (rituximab, cyclophosphamide, doxorubicin, vincristine, and prednisone) or R-CHP (omitting vincristine) was used to treat CD30-expressing high-intermediate and high-risk treatment-naïve DLBCL patients [17]. The ORR was $91 \%$, and the 18 -month progression-free survival (PFS) rate was 79\%. Although one study was initiated to compare $\mathrm{BV}+\mathrm{RCHOP}$ versus standard $\mathrm{R}-\mathrm{CHOP}$, it was terminated while ongoing on the sponsor's decision.

\section{Targeting CD19}

CD19 is expressed in the B-cell lineage at an earlier stage than that in CD20, which provides an advantage to CD19-targeted drugs over rituximab, especially for early B-cell neoplasms [18]. Blinatumomab is a bispecific T-cell engager (BiTE) that links CD19-positive B cells to CD3-positive T cells, resulting in the induction of $\mathrm{T}$-cell-mediated serial lysis of B cells. In a phase I trial, 76 RR B-cell lymphoma patients were treated with blinatumomab at a starting dose of $0.5 \mu \mathrm{g} / \mathrm{m}^{2} /$ day, and an ORR of $69 \%$ was achieved at a target dose of $60 \mu \mathrm{g} / \mathrm{m}^{2} /$ day [19]. Long-term follow-up analysis showed sustained remission in patients who had received $\geq 60 \mu \mathrm{g} / \mathrm{m}^{2} /$ day and responded as their median overall survival (OS) reached 7.7 years [20].

Tafasitamab (MOR208) is a CD19-targeting monoclonal antibody, with an engineered $\mathrm{Fc}$ region that enhances both antibody-dependent cellular cytotoxicity (ADCC) and ADCP [21]. While the drug exhibits cytotoxicity by itself [22], in the phase II L-MIND trial, lenalidomide was combined to modify the microenvironment to enhance ADCC [23]. After 81 patients with RR DLBCL received tafasitamab (12 $\mathrm{mg} / \mathrm{kg}$ ) and lenalidomide (25 $\mathrm{mg}$ once daily for 3 of $4 \mathrm{wk}$ ) for 12 months, followed by tafasitamab monotherapy until disease progression, 34 (43\%) and 14 patients (18\%) achieved complete and partial responses, respectively. The estimated median progression-free survival (PFS) was 12.1 months, and the 18 -month OS rate was $64 \%$. The combination was well tolerated, as neutropenia was the most common treatment-emergent adverse event (48\%), which was well controlled with the use of granulocyte colony-stimulating factor. Non-hematologic toxicities included skin rash (36\%), diarrhea (33\%), and asthenia (23\%). Based on these results, the FDA approved tafasitamab plus lenalidomide for the treatment of RR DLBCL in July 2020. An observational retrospective cohort study, The RE-MIND trial, analyzed patients with RR DLBCL who were treated with lenalidomide monotherapy, and those treated with tafasitamab plus lenalidomide in the L-MIND trial using estimated propensity score-based matching methodology [24]. Each arm included 76 patients, and a significantly higher ORR $(67.1 \%$ vs. $34.2 \%, P<0.0001)$ and prolonged OS (HR, 0.47; $P=0.0008$ ) were observed in the combination arm. Currently, the B-MIND trial is ongoing to compare the alkylating agent bendamustine plus rituximab (BR) versus bendamustine plus tafasitamab in RR DLBCL patients (NCT02763319).

\section{Targeting CD22}

CD22 is a regulatory component of the B-cell receptor (BCR) complex, expressed exclusively in pre-B and mature B cells. Since CD22 endocytosis can be triggered efficiently, antibodies and antibody-based immunotoxins against CD22 have been developed to treat B-cell lymphomas and leukemias.

PIV is an ADC targeting CD22 that shares the same microtubule-disrupting agent with $\mathrm{BV}$ or polatuzumab vedotin (PoV), which targets CD79b. In the ROMULUS trial, 81 patients with DLBCL and 42 patients with follicular lymphoma (FL) were recruited and randomly assigned to receive rituximab plus either PiV (R-PiV) or PoV (R-PoV) [25]. In the DLBCL cohort, the ORR for R-PiV was $60 \%$, while that for R-PoV was 54\%. In the FL cohort, the ORRs for $\mathrm{R}-\mathrm{PiV}$ and $\mathrm{R}-\mathrm{PoV}$ were $62 \%$ and $70 \%$, respectively. However, the duration of response was 2-times longer in the R-PoV arm in the DLBCL cohort (6.2 mo vs. $13.4 \mathrm{mo}$ ), and more patients in the $\mathrm{R}-\mathrm{PiV}$ arm discontinued treatment for peripheral neuropathy. As $\mathrm{PiV}$ was outperformed by $\mathrm{PoV}$, this agent is no longer in development.

Moxetumomab pasudotox is a recombinant immunotoxin that consists of an anti-CD22 immunoglobulin variable domain genetically joined to a Pseudomonas exotoxin. In a pivotal open-label study, 80 patients with RR hairy cell leukemia (HCL), who received at least two prior systemic therapies, including treatment with a purine nucleoside analog, received moxetumomab pasudotox and showed a complete response rate of $41 \%$ and an ORR of $75 \%$ [26]. Hematologic remission was observed in $80 \%$ of patients. The most frequent adverse events (AEs) were peripheral edema (39\%), nausea (35\%), fatigue (34\%), and headache (33\%). In September 2018, the FDA approved this agent for the treatment of RR HCL.

\section{Targeting CD79b}

$\mathrm{CD79b}$ is a component of the BCR. Activated B-cell-like (ABC) DLBCL depends on the BCR signaling pathway for survival, and knockdown of proximal BCR subunits, including CD79b, killed ABC DLBCLs with wild-type CARD11 [27]. Encouraged by the results from the aforementioned ROMULUS trial, the GO29365 trial compared the efficacy and safety of BR alone versus that in BR plus PoV (RBP) in RR FL and DLBCL. In the FL cohort $(\mathrm{N}=80)$, the median number of prior therapies was 2 , and the incidence of patients with progressive disease within 24 months (POD24) were $24 \%$ and $31 \%$ in the BR and RBP arms, respectively. A similar response rate $(73 \%$ vs. $77 \%)$ and median PFS $(17.3$ mo vs. $17 \mathrm{mo})$ were noted [28]. In the DLBCL cohort $(\mathrm{N}=80)$, the median number of prior therapies was 2, and 19 patients (47.5\%) for each arm were ABC DLBCL [29]. Patients in the RBP arm showed a significantly higher CR rate $(40.0 \%$ vs. $17.5 \%, P=0.026$ ), longer median PFS (9.5 mo vs. 3.7 mo, $P<0.001)$, and longer median OS (12.4 mo vs. $4.7 \mathrm{mo}$, $P=0.002)$. Although patients in the RBP arm experienced more frequent neutropenia, anemia, and thrombocytopenia, the incidence of grade 3-4 infection was similar (23.1\% vs. 
20.5\%). No relationship was observed between levels of CD79b expression and clinical outcome with the addition of PoV, but ABC DLBCL patients demonstrated higher CR rates $(47.1 \%$ vs. $26.7 \%)$, prolonged median PFS (10.76 mo vs. $2.50 \mathrm{mo}$ ), and prolonged OS (15.38 mo vs. $7.23 \mathrm{mo}$ ) compared to those in germinal center B-cell-like DLBCL patients. The GO29365 trial is the first randomized trial demonstrating an OS benefit in patients with RR DLBCL, and the FDA approved the RBP combination in June 2019. Currently, the POLARIX trial is ongoing to compare standard $\mathrm{R}-\mathrm{CHOP}$ versus PoV plus R-CHP in newly diagnosed DLBCL patients (NCT03274492).

\section{CONCLUSION}

The introduction of rituximab heralded a new era of treatment for B-cell lymphoma. For nearly 20 years, rituximab-based therapies have been the standard of care, with no other treatments surpassing this position. On the other hand, if a patient experiences relapsed or refractory disease after rituximab-based treatment, there are limited options for salvage. Although chimeric antigen receptor T-cell therapy has become a promising option for this population [30-32], its generalized use is not yet available.

In this regard, recent advances in immunochemotherapeutics deserve attention. Various mechanisms, including CD20 modulation and inhibition of CD20-mediated ADCC, have been postulated as treatments after rituximab failure [33]. Indeed, it is hoped that the discovery of other therapeutic targets (tafasitamab, polatuzumab vedotin) and enhancement of the cytotoxicity of effector cells (mosunetuzumab, addition of lenalidomide) may help to overcome rituximab resistance. Ongoing and future clinical trials give an indication of how these drugs will change the landscape of the treatment of B-cell lymphoma.

\section{Authors' Disclosures of Potential Conflicts of Interest}

No potential conflicts of interest relevant to this article were reported.

\section{REFERENCES}

1. Shaffer AL, Rosenwald A, Staudt LM. Lymphoid malignancies: the dark side of B-cell differentiation. Nat Rev Immunol 2002;2:920-32.

2. Kosmas C, Stamatopoulos K, Stavroyianni N, Tsavaris N, Papadaki T. Anti-CD20-based therapy of B cell lymphoma: state of the art. Leukemia 2002;16:2004-15.

3. Coiffier B. Rituximab therapy in malignant lymphoma. Oncogene 2007;26:3603-13.

4. Marcus R, Davies A, Ando K, et al. Obinutuzumab for the first-line treatment of follicular lymphoma. N Engl J Med 2017;377: 1331-44.
5. Crump M, Neelapu SS, Farooq U, et al. Outcomes in refractory diffuse large B-cell lymphoma: results from the international SCHOLAR-1 study. Blood 2017;130:1800-8.

6. Landsburg DJ, Falkiewicz MK, Maly J, et al. Outcomes of patients with double-hit lymphoma who achieve first complete remission. J Clin Oncol 2017;35:2260-7.

7. Casulo C, Byrtek M, Dawson KL, et al. Early relapse of follicular lymphoma after rituximab plus cyclophosphamide, doxorubicin, vincristine, and prednisone defines patients at high risk for death: an analysis from the National LymphoCare Study. J Clin Oncol 2015;33:2516-22.

8. Vitolo U, Trněný $M$, Belada $D$, et al. Obinutuzumab or rituximab plus cyclophosphamide, doxorubicin, vincristine, and prednisone in previously untreated diffuse large B-cell lymphoma. J Clin Oncol 2017;35:3529-37.

9. Bartlett NL, Wilson WH, Jung SH, et al. Dose-adjusted EPOCH-R compared with R-CHOP as frontline therapy for diffuse large B-cell lymphoma: clinical outcomes of the phase III intergroup trial alliance/CALGB 50303. J Clin Oncol 2019;37:1790-9.

10. Younes A, Sehn LH, Johnson P, et al. Randomized phase III trial of ibrutinib and rituximab plus cyclophosphamide, doxorubicin, vincristine, and prednisone in non-germinal center B-cell diffuse large B-cell lymphoma. J Clin Oncol 2019;37:1285-95.

11. Gisselbrecht C, Glass B, Mounier N, et al. Salvage regimens with autologous transplantation for relapsed large B-cell lymphoma in the rituximab era. J Clin Oncol 2010;28:4184-90.

12. Slack GW, Steidl C, Sehn LH, Gascoyne RD. CD30 expression in de novo diffuse large B-cell lymphoma: a population-based study from British Columbia. Br J Haematol 2014;167:608-17.

13. $\mathrm{Hu} \mathrm{S}, \mathrm{Xu}-$ Monette $\mathrm{ZY}$, Balasubramanyam A, et al. CD30 expression defines a novel subgroup of diffuse large B-cell lymphoma with favorable prognosis and distinct gene expression signature: a report from the International DLBCL RituximabCHOP Consortium Program Study. Blood 2013;121:2715-24.

14. Higgins JP, Warnke RA. CD30 expression is common in mediastinal large B-cell lymphoma. Am J Clin Pathol 1999; 112:241-7.

15. Pileri SA, Gaidano G, Zinzani PL, et al. Primary mediastinal B-cell lymphoma: high frequency of BCL-6 mutations and consistent expression of the transcription factors OCT-2, BOB.1, and PU.1 in the absence of immunoglobulins. Am J Pathol 2003;162:243-53.

16. Jacobsen ED, Sharman JP, Oki Y, et al. Brentuximab vedotin demonstrates objective responses in a phase 2 study of relapsed/ refractory DLBCL with variable CD30 expression. Blood 2015; 125:1394-402.

17. Budde LE, Halwani A, Yasenchak CA, et al. Results of an ongoing phase 2 study of brentuximab vedotin with $\mathrm{rchp}$ as frontline therapy in patients with high-intermediate/high-risk diffuse large B cell lymphoma (DLBCL). Blood (ASH Annual Meeting Abstracts) 2016;128(Suppl):104.

18. Carter RH, Myers R. Germinal center structure and function: lessons from CD19. Semin Immunol 2008;20:43-8.

19. Goebeler ME, Knop S, Viardot A, et al. Bispecific T-cell engager (BiTE) antibody construct blinatumomab for the treatment of patients with relapsed/refractory non-Hodgkin lymphoma: final results from a phase I study. J Clin Oncol 2016;34:1104-11.

20. Dufner V, Sayehli CM, Chatterjee M, et al. Long-term outcome 
of patients with relapsed/refractory B-cell non-Hodgkin lymphoma treated with blinatumomab. Blood Adv 2019;3:2491-8.

21. Horton HM, Bernett MJ, Pong E, et al. Potent in vitro and in vivo activity of an Fc-engineered anti-CD19 monoclonal antibody against lymphoma and leukemia. Cancer Res 2008;68:8049-57.

22. Jurczak W, Zinzani PL, Hess G, et al. A phase IIa, open-label, multicenter study of single-agent tafasitamab (MOR208), an Fc-optimized anti-CD19 antibody, in patients with relapsed or refractory B-cell non-Hodgkin's lymphoma: long-term follow-up, final analysis. Blood (ASH Annual Meeting Abstracts) 2019; 134(Suppl 1):4078.

23. Salles G, Duell J, González Barca E, et al. Tafasitamab plus lenalidomide in relapsed or refractory diffuse large B-cell lymphoma (L-MIND): a multicentre, prospective, single-arm, phase 2 study. Lancet Oncol 2020;21:978-88.

24. Zinzani PL, Rodgers T, Marino D, et al. Re-mind study: comparison of tafasitamab+lenalidomide (L-MIND) vs lenalidomide monotherapy (REAL-world data) in transplant-ineligible patients with relapsed/refractory diffuse large B-cell lymphoma. 25th EHA Congress Abstracts 2020:S238.

25. Morschhauser F, Flinn IW, Advani R, et al. Polatuzumab vedotin or pinatuzumab vedotin plus rituximab in patients with relapsed or refractory non-Hodgkin lymphoma: final results from a phase 2 randomised study (ROMULUS). Lancet Haematol 2019;6: e254-65.

26. Kreitman RJ, Dearden C, Zinzani PL, et al. Moxetumomab pasudotox in relapsed/refractory hairy cell leukemia. Leukemia 2018;32:1768-77.

27. Davis RE, Ngo VN, Lenz G, et al. Chronic active B-cell-receptor signalling in diffuse large B-cell lymphoma. Nature 2010;463: 88-92.

28. Sehn LH, Kamdar M, Herrera AF, et al. Randomized phase 2 trial of polatuzumab vedotin (POLA) with bendamustine and rituximab (BR) in relapsed/refractory (R/R) FL and DLBCL. J Clin Oncol (ASCO Annual Meeting Abstracts) 2018;36(15 Suppl): 7507.

29. Sehn LH, Herrera AF, Flowers CR, et al. Polatuzumab vedotin in relapsed or refractory diffuse large B-cell lymphoma. J Clin Oncol 2020;38:155-65.

30. Neelapu SS, Locke FL, Bartlett NL, et al. Axicabtagene ciloleucel CAR T-cell therapy in refractory large B-cell lymphoma. N Engl J Med 2017;377:2531-44

31. Abramson JS, Palomba ML, Gordon LI, et al. Lisocabtagene maraleucel for patients with relapsed or refractory large B-cell lymphomas (TRANSCEND NHL 001): a multicentre seamless design study. Lancet 2020;396:839-52.

32. Schuster SJ, Bishop MR, Tam CS, et al. Tisagenlecleucel in adult relapsed or refractory diffuse large B-cell lymphoma. N Engl J Med 2019;380:45-56.

33. Bonavida B. Postulated mechanisms of resistance of B-cell non-Hodgkin lymphoma to rituximab treatment regimens: strategies to overcome resistance. Semin Oncol 2014;41:667-77. 